\title{
Moving the Needle: Improving the Care of the Gout Patient
}

Jon Golenbiewski · Robert T. Keenan

Received: January 28, 2019 / Published online: March 2, 2019

(C) The Author(s) 2019

\begin{abstract}
Gout is a the most common inflammatory arthritis in the United States. It is a significant cause of morbidity, disability, lost work days, and high healthcare utilization due to intermittent attacks, chronic inflammation, and joint damage. Despite our understanding of the prelude and pathophysiology of gout, hyperuricemia, it is still poorly misunderstood by patients and poorly managed by healthcare providers. Several parallel treatment paradigms have been developed by professional societies around the world based on the understanding of how hyperuricemia occurs, gout
\end{abstract}

Enhanced digital features To view enhanced digital features for this article go to https://doi.org/10.6084/ m9.figshare.7706906.

J. Golenbiewski

Division of Rheumatology, Department of

Medicine, Duke University School of Medicine,

Durham, NC, USA

R. T. Keenan

Duke Gout and Crystal Arthropathies Clinic,

Durham, NC, USA

R. T. Keenan

Duke Specialty Infusion Centers, Durham, NC, USA

R. T. Keenan $(\bowtie)$

Division of Rheumatology and Immunology,

Department of Medicine, Duke University School of

Medicine, Durham, NC, USA

e-mail: robert.keenan@duke.edu epidemiology, expert opinion, and clinical trials data in order to lower uric acid and eventually eliminate the patient's crystal burden. This review focuses on both the treatment of acute attacks, and more importantly, the long-term management of gout and the lowering of serum uric acid levels to a goal of $<6 \mathrm{mg} / \mathrm{dl}$ $(0.360 \mathrm{mmol} / \mathrm{l})$ or treating to target. Treating to a target serum uric acid goal is an opportunity to decrease morbidity and improve the quality of care of every gout patient.

Keywords: Allopurinol; Colchicine; Febuxostat; Gout; Lesinurad; Pegloticase; Prophylaxis; Treatment

\section{INTRODUCTION}

Gout is a severe, often debilitating inflammatory arthritis caused by deposition of monosodium urate crystals in the joint space, periarticular structures and soft tissues, producing a pronounced inflammatory response in the patient experienced as pain. Although the disease is well understood and effective treatment options are available, gout is often poorly managed by both primary care physicians and rheumatologists alike [1]. Large retrospective studies show poor prescription rates of urate lowering therapy (ULT) and high incidence of failure to reach appropriate serum uric acid 
(SUA) levels, consequently resulting in poor disease control [1-4]. Inadequately treated gout leads to recurrent attacks, chronic inflammation, joint damage, subclinical crystal deposition, and tophi formation, which can result in limited activity, dependence on others, recurrent work absences, decreased work productivity, and high rates of healthcare utilization [5-7].

Given the negative impact of disease on the individual patient and society, and its increasing prevalence and world-wide reach, education regarding best practices in gout management is of paramount importance. In this paper, we define the problem of gout through epidemiologic review, discuss uric acid metabolism and the pathophysiology of gout, and present treatment recommendations for the acute flare and chronic disease, with a focus on the concept of treat-to-target with urate-lowering therapy.

This article is based on previously conducted studies and does not contain any studies with human participants or animals performed by any of the authors.

\section{Incidence/Prevalence}

Gout is a worldwide disease of increasing prevalence, currently in the range of $1-4 \%$, and exceeding more than $10 \%$ in some Oceanic countries [8] [9]. In the United States alone, the estimated overall prevalence of gout among adults corresponds to 9.2 million people [10]. Along with prevalence, the burden of gout is increasing around the world as well, supported by an increase in gout disability-adjusted life years from 76,000 in 1990 to 114,000 in 2010 [11]. The incidence of gout increases with age and is more common in men, with the male-tofemale ratio generally along the order of 3 to 4:1; however, this gap closes with aging [9].

Gout should not be viewed simply as a disease affecting the joints, but rather of multiple organ systems, as patients with gout frequently have comorbidities including hypertension, cardiovascular disease (CVD), renal impairment, obesity, hyperlipidemia, and diabetes and the metabolic syndrome [12]. According to the
2007-2008 National Health and Nutrition Examination Survey data (NHANES), $74 \%$ of participants with gout had hypertension, 71\% had stage 2 or greater chronic kidney disease, $53 \%$ were obese, $26 \%$ had diabetes, $14 \%$ had a history of myocardial infarction, and $10 \%$ had a history of stroke [13]. Furthermore, large prospective studies have shown that gout is associated with an increased risk of death, primarily due to cardiovascular disease $[8,14]$.

\section{Mechanism}

Urate is the byproduct of purine degradation in the body, and therefore depends both on intrinsic production and dietary intake, with the greater part derived from endogenous liver synthesis, and the lesser being consumption of purine-rich foods [6]. Humans lack uricase, and therefore rely on other mechanisms of elimination to maintain a normal total body purine balance. Urate is eliminated primarily by the kidneys, which accounts for approximately twothirds of urate excretion [8], and gastrointestinal elimination plays a smaller but important role.

The balance between hepatic production of urate and intestinal or renal urate excretion pathways determines an individual's serum urate levels [15]. Hyperuricemia more commonly results from decreased renal urate excretion (underexcretors), but can also be ascribed to excessive urate production, or a combination of both processes [6]. Urate-associated loci have been identified through genome-wide association studies (GWAS) that contain genes encoding renal and gut uric acid transporters, and with this recognition, genetic contributions may be playing a larger role in hyperuricemia and gout than previously appreciated $[15,16]$.

Hyperuricemia, defined as a serum urate level of $6.8 \mathrm{mg} / \mathrm{dl}(0.360 \mathrm{mmol} / \mathrm{l})$ or greater, is the central risk factor for developing gout [17]. Uric acid is a weak organic acid that exists predominantly as monosodium urate (MSU) monohydrate at physiologic $\mathrm{pH}$ (7.4). At concentrations greater than $6.8 \mathrm{mg} / \mathrm{dl}$ $(0.360 \mathrm{mmol} / \mathrm{l})$, MSU exceeds its limit of 
solubility in serum under physiologic conditions, allowing precipitation and the formation of MSU crystals [6].

The presence of MSU crystals, mediated by interaction with resident macrophages, leads to an inflammatory cascade involving IL-1B production, activation of neutrophils and mast cells, activation of the NLRP3 inflammasome, and release of pro-inflammatory cytokines such as IL-6 [6]. Acute attacks of gout are often triggered by specific events such as trauma, surgery, intercurrent illness, excess alcohol intake or drugs that alter serum urate levels [18]. Such events may stimulate de novo formation of MSU crystals or may trigger release of microcrystals from existing crystal deposition within the joint $[18,19]$.

The progression of gout can be defined by four pathophysiological stages: hyperuricemia without evidence of monosodium urate crystal deposition or gout; crystal deposition without symptomatic gout; crystal deposition with acute gout flares; and advanced gout characterized by tophi, chronic gouty arthritis, and radiographic erosions [20]. The natural history of an acute gouty attack, without treatment, typically lasts 7-10 days. The first presentation is often defined by podagra (gouty inflammation of the big toe), but can also involve the feet, ankle, knees, hands, wrists, or elbows. An acute attack is followed by complete resolution in signs and symptoms of joint inflammation during the so-called intercritical period [8], which can last for a variable amount of time. The course of gout following the first attack is highly variable, with some patients having no recurrence, while in other individuals the flares become more frequent, leading to advanced gout with chronic pain, joint destruction, and tophus formation [1].

\section{Treatment}

The differential diagnosis of an acute gout flare includes infection, other forms of inflammatory arthritis (reactive arthritis or rheumatoid arthritis, for example), and alternative crystalline arthropathies such as calcium pyrophosphate deposition disease (CPPD).
Therefore, the diagnosis of gout should be made with crystal confirmation from analysis of synovial or bursal fluid or tophus aspiration whenever possible. If there is clinical concern for infection, aspiration with cell count, Gram stain, and culture should be performed.

A low or normal SUA in the setting of an acute flare cannot be used to rule out the diagnosis of gout, as SUA may be falsely lowered secondary to the uricosuric effect of ACTH and cortisol released in response to pain, in addition to the indirect uricosuric effect of IL- 6 on the kidney [21-24]. Imaging modalities, including musculoskeletal ultrasound that reveals the double contour sign, and dual-phase CT are alternative ways of supporting the diagnosis when crystal evidence is not possible (Fig. 1).

\section{Acute Flare}

Management of acute gout flare is aimed at relieving pain through rapid control of inflammation and restoring function. Therapy should be initiated as soon as possible at the first sign of an attack [25-27], as this improves the likelihood of a positive response to therapy, and potentially minimizes the dose and duration of potentially harmful medications. Accordingly, the American College of Rheumatology (ACR) recommends providing patients with education and instructions to initiate treatment of an acute flare at home [25]. As a general rule, uratelowering therapy should be continued as prescribed during an acute flare, unless an absolute contraindication exists. This promotes compliance with treatment, mitigates the risk of failing to resume therapy after symptom resolution, and minimizes changes in SUA, as this can lead to continued or recurrent flares.

The specific anti-inflammatory treatment utilized depends on the severity of presentation, number of joints involved, the presence of medical comorbidities, and concomitant pharmacologic agents being used. NSAIDs, colchicine, or prednisone are the most effective means to control the acute flare, and all can be considered first-line therapy. Combination therapy with any of the above may also be necessary for severe, polyarticular flares not responding to a 


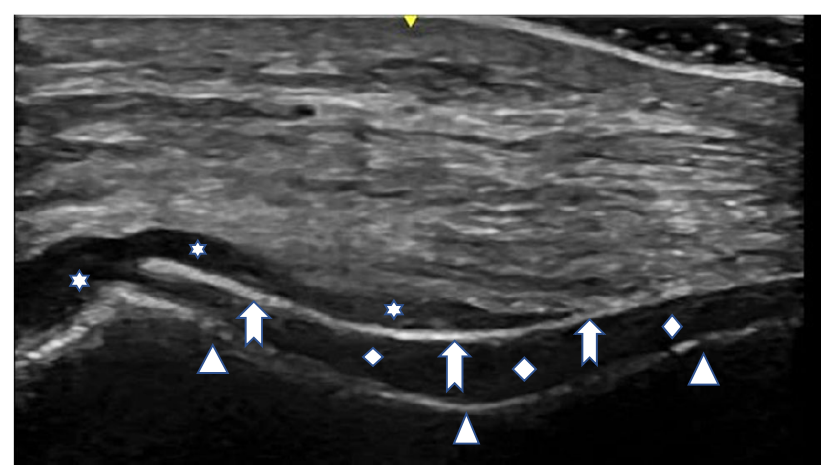

Fig. 1 Double contour sign on musculoskeletal ultrasound of the knee. Notched arrow: uric acid crystal deposition; arrowhead: tibial plateau; diamond: meniscus; star: small effusion

single agent, or historically fail to respond to monotherapy [25]. Corticosteroid injections are an appropriate treatment option for patients with involvement limited to only one or two joints and may be an ideal choice in patients with contraindications to systemic therapy, or with multiple comorbid conditions that limit systemic therapeutic options [28]. If the patient does not respond to initial therapy (and the diagnosis of gout is not in question), changing to an alternative agent (such as from colchicine to an NSAID, for example), or adding another agent in combination may be warranted $[25,26]$.

While there are only three FDA approved NSAIDs (indomethacin, naproxen, sulindac), there are many to select from in the treatment of acute gout. The specific NSAID chosen is less important than the dose and duration of treatment. NSAIDS should be prescribed at the full anti-inflammatory dose for the shortest period of time for the duration of the flare [8]. The authors typically use naproxen $500 \mathrm{mg}$ BID, given its less frequent dosing regimen, or ibuprofen $800 \mathrm{mg}$ TID, for example. Lower doses of NSAIDS have less of an anti-inflammatory effect, potentially prolonging the duration of flare, leading to persistent pain and disability.

Prior to prescribing NSAIDS, a review of comorbidities and medications should be performed to see if the patient is an appropriate candidate for therapy. Reasons to consider avoiding NSAIDS include the presence of chronic kidney disease, coronary artery disease, heart failure, peptic ulcer disease, hepatic disease, and history of gastrointestinal intolerance. The presence of these conditions should not automatically disqualify use, but should lead to a benefit-versus-risk assessment and discussion with the patient. A medication review should be performed to look for potential harmful interactions. The combination of an NSAID and an ACE inhibitor is of particular concern because of the combined effects on blood pressure and renal function [12]. Additionally, concurrent use of anticoagulation or other antiplatelet agents and the resulting bleeding risk need to be considered, and often times negate the use of NSAIDS.

Colchicine is an anti-inflammatory agent that serves as another primary treatment option in the management of acute gout. It works through multiple proposed mechanisms, including microtubule inhibition resulting in decreased NLRP3 inflammasome activation [29]. In the US, colchicine is typically given as a one-time dose of $1.2 \mathrm{mg}$, followed by $0.6 \mathrm{mg} 1 \mathrm{~h}$ later, with daily dosing starting as soon as $12 \mathrm{~h}$ thereafter until flare resolution. Colchicine is felt to be more effective when initiated early in the setting of a flare. Consistent with this view, The European League Against Rheumatism (EULAR) advocates for the 'pill in the pocket' approach to treat flares in fully informed patients [27], and recommends treatment with colchicine within $12 \mathrm{~h}$ of flare onset, although practically speaking, colchicine should be taken at the very first sign of a flare. 
Colchicine has known side effects of diarrhea and myopathy. It also has the potential to cause agranulocytosis. The dose should be reduced in patients with kidney impairment and those receiving cytochrome P450 3A4 inhibitors such as diltiazem, verapamil, or p-glycoprotein inhibitors such as cyclosporine, and avoided in patients taking clarithromycin. Colchicine should also be used with caution in those with liver disease or taking statins [8].

Steroids are a viable option in the treatment of acute gout, given their potent anti-inflammatory effects. They can be given orally, intravenously, or as an intramuscular depot. IV steroids are often utilized for inpatient treatment of a severe, polyarticular flare. A typical effective prednisone regimen is dosed at a minimum of $0.5 \mathrm{mg} / \mathrm{kg} /$ day for the duration of the flare. Sometimes, initial treatment with $0.5 \mathrm{mg} / \mathrm{kg} /$ day, followed by a prednisone taper for a total treatment duration approaching 14 days, is necessary due to recurrent flares with steroid discontinuation. If there are no contraindications, the authors typically start with $50 \mathrm{mg}$ daily and taper by $10 \mathrm{mg}$ every $2-3$ days based on response, for a course lasting 10-14 days. Shorter courses increase the risk of a rebound flare.

Hyperglycemia resulting from steroid use is a valid concern in the management of gout as insulin resistance and hyperglycemia often accompany disease. Many patients with gout have advanced renal impairment, in which NSAID or colchicine use is contraindicated and steroid use is unavoidable. Increases in blood sugars can be managed in the short term while the acute episode is treated [12], and patients with diabetes should be educated on monitoring blood glucose levels and may require temporary adjustments to their hypoglycemic regimen.

Canakinumab and anakinra are IL-1 inhibitors used for cases of refractory gout or when alternative therapies cannot be used. It exerts its effect through inhibition of the NLRP3 inflammasome and attenuation of the accompanying inflammatory cascade. Canakinumab is a subcutaneous injection given once every 8 weeks. Anakinra is available in a subcutaneous form that is typically given for a duration of 3-5 days.
It is highly effective, providing rapid improvement in pain and swelling, with the most common side effect being injection site pain. IL1 inhibitors such as anakinra are often utilized in the inpatient setting mainly when NSAIDS, colchicine, and corticosteroids are contraindicated. There are several case series in the literature demonstrating the efficacy and safety of anakinra in such complicated hospitalized patients [30]. Anakinra is currently being evaluated in phase II studies for its use in the outpatient setting [31]. Canakinumab is approved for use in acute gout in Europe, but not in the U.S. Finally, adrenocorticotropic hormone (ACTH) is also an effective treatment for acute gout, but is cost-prohibitive in the U.S.

\section{Urate-Lowering Therapy}

\section{When to Treat}

While the risk of developing gout increases with rising serum urate concentrations, not all individuals with elevated SUA will develop gout [17]. Accordingly, treatment of asymptomatic hyperuricemia is not recommended currently in the United States and Europe. Indications for ULT include the presence of tophus or tophi (verified by clinical examination or imaging study), two or more flares per year, or one flare in the setting of chronic kidney disease stage 2 or worse and history of urolithiasis per the ACR 2012 guidelines [32] (Fig. 2). The EULAR makes similar recommendations, and further suggests proceeding with treatment close to the time of the first diagnosis in young patients $(<40$ years), or with a very high SUA level ( $>8 \mathrm{mg} / \mathrm{dl}$ or $>0.475 \mathrm{mmol} / \mathrm{l})$, and/or comorbidities such as renal impairment, hypertension, ischemic heart disease, and heart failure [27]. The British Society for Rheumatology Guidelines share the same overarching principles as ACR and EULAR, and also recommends consideration of extending therapy to those with gout taking diuretics and those developing gout at a young age, as these patients are more likely to experience a higher cumulative burden of disease over the course of many years, and also to have a heritable cause that predisposes to a lifetime of active disease [26]. 

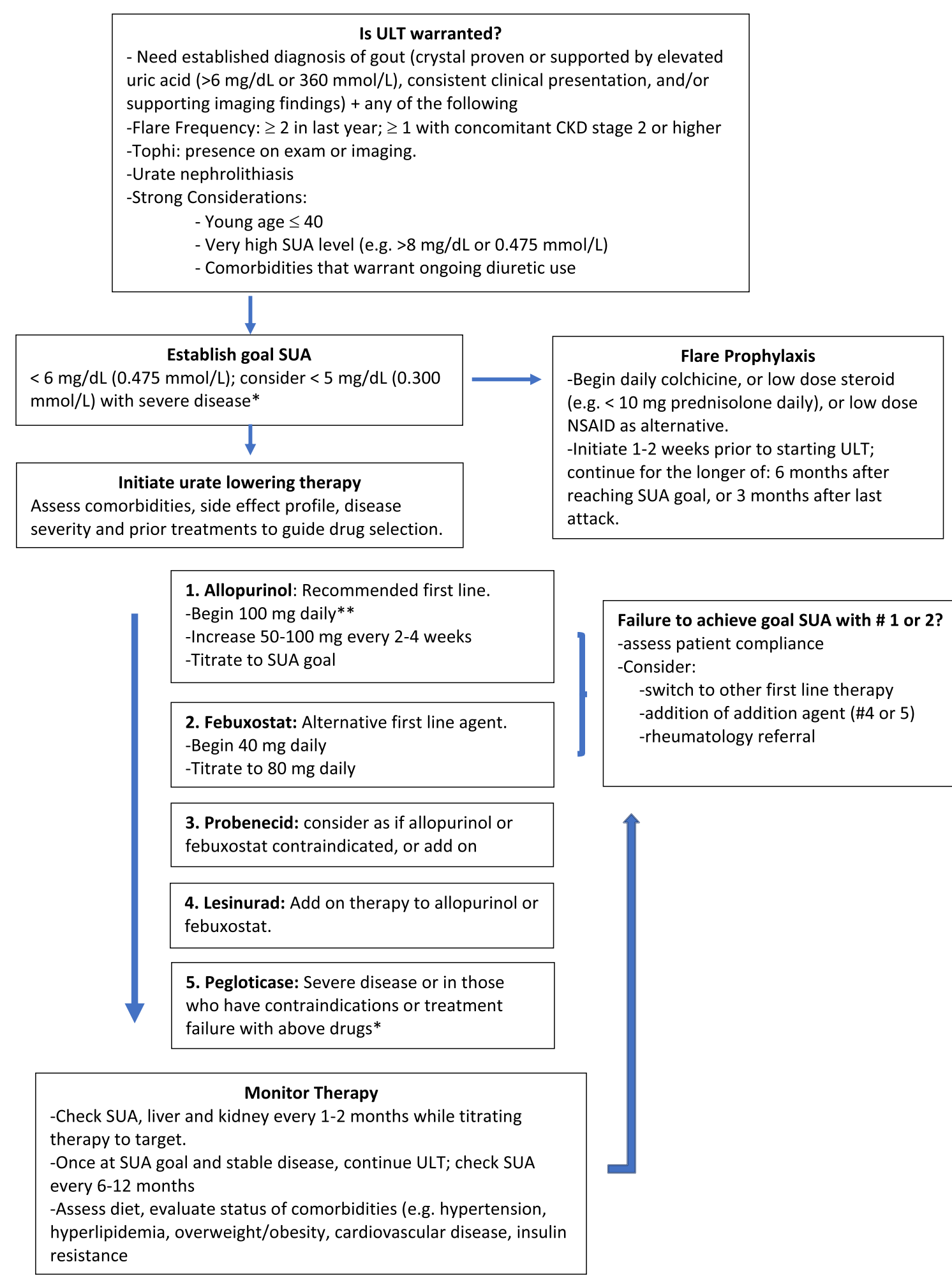

Fig. 2 Gout treatment algorithm utilizing T2T approach. *Severe disease: tophaceous disease, chronic arthropathy, frequent attacks. Consider decreasing goal to $6 \mathrm{mg} / \mathrm{dl}$
$(0.360 \mathrm{mmol} / \mathrm{l})$ once $\mathrm{UA}$ at goal and disease stable. ${ }^{* *}$ Consider starting lower based on creatinine clearance 
There is some controversy regarding whether or not to start ULT during an acute gout flare, such as in a hospital setting. Traditionally, starting ULT during a flare was thought to potentially exacerbate or prolong an acute flare, and ULT should be delayed until resolution. Recently, there have been small studies that showed starting ULT during an acute flare while being treated did not necessarily worsen or prolong a flare, but rather was a window of opportunity to start ULT in a hospital setting [33]. Given there is not good evidence to support either starting ULT during a flare or waiting until resolution [34], it may need to be a clinical judgment. In our practice, we typically wait until resolution of the flare before starting ULT, although we may make exceptions in the hospital setting and start before full resolution occurs to ensure initiation of ULT and tolerance prior to discharge.

\section{Non-pharmacologic Management}

Non-pharmacologic management is beneficial in all patients with gout and should focus on education, diet, and lifestyle recommendations, as well as screening for comorbidities that occur with gout [26, 27, 32]. Knowledge gaps frequently exist in understanding basic gout principles, including the use of ULT, optimal goal SUA levels, appropriate duration of ULT, lifestyle modifications, and the use of prophylaxis for gout flares during the early phase of uratelowering therapy [35]. It is important to ensure the patient possesses a solid understanding of the underlying pathogenesis of gout, in addition to the rationale for long-term ULT in order for treatment to be successful $[8,36]$.

In general, diet should be considered an adjunct to urate-lowering therapy, as diet alone often fails in adequately treating gout, and can lead to delayed treatment with urate-lowering pharmacologic therapy. The primary aims of diet and lifestyle modifications in gout are the promotion and maintenance of health and preventative care through optimal management of comorbidities that are commonly observed with gout, including coronary artery disease, obesity, metabolic syndrome, diabetes mellitus, hyperlipidemia, and hypertension [32]. Diets that promote weight loss can reduce serum uric acid levels [36], and also have beneficial effects on related comorbidities (e.g., improvement in lipid profile, blood glucose levels, and lower blood pressure). Many foods are associated with increased serum uric acid levels including red and processed meats, shellfish, alcoholic beverages, sugar-containing beverages and foods (including high-fructose corn syrup), and refined grains $[15,36,37]$. These should be consumed in moderation or avoided altogether, in some cases.

Certain diets, including the Dietary Approaches to Stop Hypertension (DASH diet) and the Mediterranean diet have been shown to reduce serum urate levels and the risk of gout [15], and are an appropriate preventative approach for those with hyperuricemia at increased risk of developing gout [37]. While diet is an important modifiable factor, it may have less of a direct role in modulating SUA levels than previously thought. A recent meta-analysis concluded that individual food items and estimates of dietary patterns explain very little variation in serum urate levels, with genetic variants explaining substantially more of the variation [15]. Nevertheless, we should still promote overall health through diet, and utilize it in conjunction with urate-lowering therapy in minimizing the burden of disease. It is important for both the provider and the patient to understand that diet alone typically does not resolve hyperuricemia, and should not delay ULT initiation.

\section{Pharmacologic Management}

\section{Treat-to-Target}

Crystal formation is reversible, which means that gout can be "cured" [1]. As such, the goal of therapy is rather simple: to lower SUA below the limit of solubility, and sometimes well below [1]. Given the fact that diet alone may not be effective in the management of gout, initiation of urate-lowering therapy is the primary means of treatment. Treat-to-target (T2T) is a concept developed for use in a number of rheumatologic diseases to guide treatment toward a predefined therapeutic level. This approach is recommended by the ACR, EULAR, and the British 
Society of Rheumatology (BSR) in the management of gout. The American College of Physicians (ACP) is the only organization that does not recommend a T2T approach [38], although their reasoning is not clear given the known pathophysiology and published evidence that surround this approach.

While the optimal SUA level has not been precisely defined, data from phase III and openlabel extension studies of ULT trials support an association between lower, sub-saturation serum urate concentrations $(<6.8 \mathrm{mg} / \mathrm{dl}$ or $<0.360 \mathrm{mmol} / \mathrm{l}$ ) and less flares, reduced tophus size, and improved morbidity [39-41]. Furthermore, there is imaging support for a T2T approach in the management of gout. In one case series, achieving a SUA level below $6 \mathrm{mg} / \mathrm{dl}$ $(0.360 \mathrm{mmol} / \mathrm{l})$ resulted in resolution of the double contour sign over time, an ultrasound finding indicative of MSU crystal deposition in cartilage that is often present in gout, whereas failure to reach a target SUA resulted in persistence of this finding [42].

Some of the major, high-quality trials used in forming the ACP guidelines were based on 12 months of trial data. It would not be unexpected to see no difference using a T2T approach in this time frame, given: (1) Despite attaining a serum target urate level, gout can still remain active in the presence of a high crystal burden, and (2) initiation of urate-lowering therapy can lead to gout flares in the short term, which would unfavorably reflect upon the intervention group [40].

Some recent trials have investigated specifically a treat-to-target intervention, which have yielded positive results. A RCT in the UK compared a nurse-led gout care T2T model to general practitioners over a 2-year period, which resulted in a high percentage of the nurse-led group achieving the recommended SUA target $(95 \%$ less than $6 \mathrm{mg} / \mathrm{dl}$ or $0.360 \mathrm{mmol} / \mathrm{l} \mathrm{com-}$ pared to $30 \%$ in the usual care group), and a significant improvement in flare frequency, presence of tophi, and quality of life compared to those in the usual-care group [43]. Additionally, a pharmacist-led T2T intervention has also shown benefit, but to a lesser degree [44]. $\mathrm{T} 2 \mathrm{~T}$ is furthermore supported by the open-label extension (OLE) study [45], which provided evidence of the efficacy of sustained ULT in patients from two replicate 6-month randomized controlled pegloticase trials [46]. Serum uric acid levels remained $<6 \mathrm{mg} / \mathrm{dl}$ $(0.360 \mathrm{mmol} / \mathrm{l})$ in a large number of previously determined pegloticase responders throughout the study and were accompanied by sustained and progressive improvements in tophus resolution, flare incidence, and quality of life.

\section{Urate-lowering Therapy}

Although patient adherence to ULT is often blamed for poor gout management, it should not be just considered a 'patient' issue. There are a number of ways to improve medication compliance with patients through regular SUA monitoring [47], and helping the patient understand the mechanism by which gout occurs. Once ULT is started, serum urate levels should be monitored monthly to every other month while titrating therapy to the target goal (Fig. 2). Once the target has been achieved, this interval can be decreased to every 6 months $[8,32,48]$. Periodic testing serves the purpose of ensuring treatment adherence, given the natural tendency for patients to de-escalate or stop treatment due to clinical stability. When patients receive inappropriately low doses of ULT, ongoing urate deposition occurs leading to progression of tophaceous deposits, further joint damage, and functional disability. It is felt that the costs of measuring serum urate are minimal, especially when compared with the damage that can occur over time with active disease, requiring the use of more aggressive and expensive therapies [39].

The ACR and EULAR recommend a goal SUA of $<6 \mathrm{mg} / \mathrm{dl}(0.360 \mathrm{mmol} / \mathrm{l})$, at the very least, based on high-quality evidence. There are circumstances where a goal of less than $5 \mathrm{mg} / \mathrm{dl}$ $(0.300 \mathrm{mmol} / \mathrm{l})$ is recommended, including in tophaceous gout (both ACR and EULAR), or severe disease as judged by chronic arthropathy, or frequent attacks (EULAR). In contrast, the BSR recommends a more stringent target of less than $5 \mathrm{mg} / \mathrm{dl}(0.300 \mathrm{mmol} / \mathrm{l})$ for everyone, but does allow for a less strict target after years of stable disease. Both the BSR and EULAR 
Table 1 Dosing and cautions of urate-lowering therapy

\begin{tabular}{|c|c|c|c|c|}
\hline Agent & MOA & Dosing & Caution & Other \\
\hline Allopurinol & $\begin{array}{l}\text { Xanthine } \\
\text { oxidase } \\
\text { inhibitor }\end{array}$ & $\begin{array}{l}100 \mathrm{mg} \text { by mouth daily, titrate } \\
\text { by } 100 \mathrm{mg} \text { every } 2-4 \text { weeks } \\
\text { to max of } 800 \mathrm{mg} \text { qd } \\
50 \mathrm{mg} \text { PO qd, titrate by } 50 \mathrm{mg} \\
\text { every } 4 \text { weeks for CKD stage } \\
\text { IV or greater }\end{array}$ & $\begin{array}{l}\text { Avoid use with } \\
\text { azathioprine or } \\
\text { mercaptopurine use } \\
\text { Hepatic impairment } \\
\text { Renal impairment: In } \\
\text { general, start low, } \\
\text { titrate dose slowly and } \\
\text { educate on symptoms } \\
\text { of AHS } \\
\text { Agranulocytosis }\end{array}$ & $\begin{array}{l}\text { Check for HLA-B*5801 allele } \\
\text { in patients of Han Chinese } \\
\text { or Tai descent to mitigate } \\
\text { risk of AHS } \\
\text { Monitor CBC, hepatic and } \\
\text { renal function at baseline, } \\
\text { then every } 4-8 \text { weeks }\end{array}$ \\
\hline Febuxostat & $\begin{array}{l}\text { Xanthine } \\
\text { oxidase } \\
\text { inhibitor }\end{array}$ & $\begin{array}{l}40-80 \mathrm{mg} \text { by mouth daily } \\
\text { (start } 20-40 \mathrm{mg})\end{array}$ & $\begin{array}{l}\text { Avoid use with } \\
\text { azathioprine or } \\
\text { mercaptopurine use } \\
\text { Caution in severe } \\
\text { hepatic impairment }\end{array}$ & $\begin{array}{l}\text { Not studied in Child-Pugh } \\
\text { Class C hepatic impairment } \\
\text { Check CBC and LFTs at } \\
\text { baseline and every } \\
8-12 \text { weeks } \\
\text { Max dose } 40 \text { mg qd CrCl } \\
<30 \text {; unknown dosing for } \\
\text { hemodialysis } \\
120 \text { mg qd approved in EU }\end{array}$ \\
\hline Probenecid & $\begin{array}{l}\text { Uricosuric } \\
\text { agent } \\
\text { (inhibits } \\
\text { proximal } \\
\text { tubule urate } \\
\text { reabsorption) }\end{array}$ & $\begin{array}{l}500 \mathrm{mg} \text { by mouth twice daily } \\
\text { (initiate } 250 \mathrm{mg} \text { twice } \\
\text { BID } \times 7 \text { days) as } \\
\text { monotherapy or } \\
\text { combination therapy with } \\
\text { XOI }\end{array}$ & $\begin{array}{l}\text { NSAID use } \\
\text { G6PD deficiency } \\
\text { Renal insufficiency } \\
\text { (avoid if } \mathrm{CrCl}<50) \\
\text { Nephrolithiasis (avoid if } \\
\text { chronic urate stones) } \\
\text { PUD } \\
\text { Blood dyscrasia }\end{array}$ & $\begin{array}{l}\text { Can cause increase in } \\
\text { concentration of } \\
\text { methotrexate, beta-lactam } \\
\text { antibiotics, numerous other } \\
\text { drugs that may require dose } \\
\text { adjustments }\end{array}$ \\
\hline Lesinurad & $\begin{array}{l}\text { URAT1 and } \\
\text { OAT4 } \\
\text { inhibitor }\end{array}$ & $\begin{array}{l}200 \mathrm{mg} \text { by mouth daily in } \\
\text { combination with } \\
\text { allopurinol } \\
\text { (doses }>300 \mathrm{mg} / \text { day) or } \\
\text { febuxostat }\end{array}$ & $\begin{array}{l}\text { Renal insufficiency }(\mathrm{d} / \mathrm{c} \\
\text { if } \mathrm{CrCl} \text { persistently } \\
<45)\end{array}$ & $\begin{array}{l}\text { Check creatinine at baseline, } \\
\text { then periodically } \\
\text { No hepatic dosing } \\
\text { recommendations } \\
\text { Acute renal failure has } \\
\text { occurred when used as } \\
\text { monotherapy }\end{array}$ \\
\hline
\end{tabular}


Table 1 continued

\begin{tabular}{|c|c|c|c|c|}
\hline Agent & MOA & Dosing & Caution & Other \\
\hline Pegloticase & $\begin{array}{l}\text { Recombinant } \\
\text { urate oxidase }\end{array}$ & $\begin{array}{l}8 \text { mg intravenously every } \\
2 \text { weeks }\end{array}$ & $\begin{array}{l}\text { Do not administer with } \\
\text { concurrent urate- } \\
\text { lowering therapy } \\
\text { Risk of anaphylactic } \\
\text { reaction with infusion } \\
\text { due to development of } \\
\text { anti-drug antibodies } \\
\text { can occur }\end{array}$ & $\begin{array}{l}\text { Should be prescribed only by } \\
\text { physicians experienced in } \\
\text { the management of } \\
\text { advanced gout }\end{array}$ \\
\hline
\end{tabular}

$C r C l$ creatinine clearance, $U R A T$ urate transporter, $O A T$ organic anion transporter

guidelines advise against maintaining serum urate at very low levels for a prolonged period of time based on the results of observational studies showing a negative association between serum urate and the risk of certain neurodegenerative diseases, although this association is still not entirely clear [40]. There are times, however, where it is appropriate to have SUA levels less than $3 \mathrm{mg} / \mathrm{dl} \quad(0.180 \mathrm{mmol} / \mathrm{l})$, including in cases of severe tophaceous disease to hasten crystal dissolution.

Given that fluctuations in uric acid can induce flares, pharmacologic prophylaxis should be provided during the urate-lowering process, which may lead to improved ULT adherence. At least 6 months of prophylactic therapy is recommended by the ACR, EULAR, and BSR. Daily to twice-daily colchicine, lowdose NSAID, or low-dose steroid $(<10 \mathrm{mg}$ day prednisolone) should be used as prophylaxis [25]. Treatment should be continued if active disease remains, as judged by the presence of tophi, recent flare, chronic gouty arthritis, or serum urate targets that are not achieved. The ACR recommends continuing prophylaxis for at least 6 months after achieving target SUA in patients, or 3 months after the last attack, whichever is longer.

There are three potential pharmacologic mechanisms for achieving target SUA: (1) inhibition of urate production through the use of xanthine oxidase inhibitors (XOI), (2) increasing renal uric acid excretion through the use of uricosuric agents, and (3) metabolism of urate to the more water-soluble and readily excretable allantoin through use of recombinant uricase [5] (Table 1). In general, the approach to ULT is to start low and go slow, although there are some instances where more aggressive therapy is warranted, mainly in severe disease requiring rapid lowering of SUA through the use of pegloticase.

Xanthine oxidase inhibitors (XOI) are the main class of medications used in the treatment of gout. Xanthine oxidase is an enzyme that through the use of oxygen, converts hypoxanthine and xanthine to urate [6]. Allopurinol is a purine analogue XOI and febuxostat is a nonpurine inhibitor of XOI; both are recommended as first-line ULT [32]. Allopurinol is inexpensive and generally well tolerated. Side effects can include elevated transaminases and myelosuppression. There is a significant interaction between xanthine oxidase inhibitors and azathioprine, which can result in profound myelosuppression [12]; this is something to consider in patients who have undergone organ transplantation or have a concurrent rheumatologic disease that requires the use of azathioprine.

Allopurinol can cause a mild form of hypersensitivity with maculopapular eruption to more severe cutaneous adverse reactions including Stevens-Johnson syndrome/toxic epidermal necrolysis (SJS/TEN), drug reaction with eosinophilia and systemic symptoms (DRESS), and allopurinol hypersensitivity syndrome (AHS) [49]. AHS is a rare but potentially 
fatal condition that occurs in $0.1 \%$ of patients and is characterized by a rash combined with eosinophilia, leukocytosis, fever, hepatitis, and progressive renal failure [49]. AHS is thought to be associated with renal impairment, diuretic use and higher starting doses of allopurinol, and occurs usually within weeks to months after starting treatment, with most cases occurring by 3 months and $90 \%$ by 6 months $[49,50]$. The HLA-B*58:01 allele is associated with an increased risk of allopurinol-induced severe cutaneous adverse reactions, and should be checked in certain Asian groups [32].

In order to minimize adverse events, it is important to initiate therapy at a low dose and titrate gradually when lowering serum uric acid. The starting dose of allopurinol should be no greater than $100 \mathrm{mg} /$ day in all patients, with the exception being those with stage 4 CKD and beyond; these patients should initiate allopurinol at no more than $50 \mathrm{mg} /$ day, titrating up gradually [32]. In our experience, it may be prudent to start allopurinol at a dose of $50 \mathrm{mg} /$ day in patients with moderate chronic kidney disease, in addition to those patients who are more sensitive and prone to flares with even small fluctuations in SUA levels.

Given safety concerns, allopurinol is often prescribed based on calculated creatinine clearance recommended dosing, which often leads to failure to achieve target SUA [51]. However, studies have shown that in patients already tolerating allopurinol, increasing doses above creatinine clearance recommended dosing is effective and safe in reaching UA goals [51-53]. In these trials, there were numerically more SAEs with higher doses, though these were not deemed allopurinol-related, and there were no cases of AHS [54]. The ACR recommends that the maintenance dosage of allopurinol can be raised above $300 \mathrm{mg}$ per day, even in those with renal impairment, provided there is adequate patient education and regular monitoring for drug hypersensitivity with symptoms such as pruritus, rash, and lab findings of elevated transaminases and peripheral eosinophilia.

Febuxostat is a hepatically metabolized, nonpurine xanthine oxidase inhibitor [50] that is also considered a first-line agent in the treatment of gout. Practically speaking, however, it is usually prescribed in cases where patients do not respond to or cannot tolerate allopurinol. Febuxostat does not offer the same titration potential as allopurinol, being available in $40 \mathrm{mg}$ and $80 \mathrm{mg}$ doses in the U.S., but is a more potent XOI when compared to allopurinol [55]. Large, high-quality trials have demonstrated the efficacy of febuxostat as compared to allopurinol in reaching target SUA goals with a favorable safety profile, including those with mild-tomoderate renal impairment $[56,57]$.

The cardiovascular safety of febuxostat has been called into question based on a number of trials showing a possible increased risk of adverse cardiac events. The CARES trial in 2018 was performed to further assess this risk. It found that among gout patients with coexisting cardiovascular disease, febuxostat was noninferior to allopurinol with respect to rates of composite endpoint major cardiovascular events, but did have higher all-cause mortality resulting from cardiovascular deaths (sudden cardiac death) [58]. There were a number of issues with this study, including high rates of treatment discontinuation and loss to followup, a paucity of information on the severity gout, lack of a placebo (which may show that allopurinol confers a safety benefit, while febuxostat may confer normal baseline risk), and the fact that all-cause deaths occurred while patients were not taking drugs $85 \%$ of the time [59]. For these reasons, in addition to the potential cardiovascular risks associated with gout and hyperuricemia, practice patterns have not changed in regards to febuxostat at this time.

Probenecid and lesinurad are agents that decrease uric acid reabsorption in the proximal tubule, thereby promoting uric acid excretion and lowering serum urate levels. Probenecid is a uricosuric agent that inhibits URAT1, OAT1, and OAT3 transporters and is considered an alternative first-line pharmacologic ULT [32]. However, it is more commonly used in combination with a XOI for added urate-lowering effect. Probenecid should be avoided in patients with a CRCL $<50$ and/or history of nephrolithiasis, as it may contribute to the formation of renal stones. Lesinurad is the newest uricosuric agent that exerts its effects 
predominately through URAT1 along with some OAT4 transporter inhibition. Lesinurad is only to be given as combination therapy due to an increased risk of renal toxicity when given as monotherapy, and should not be given if the eGFR is $<45 \mathrm{ml} / \mathrm{min}$ per FDA labeling [60]. Adequate hydration of at least 21 /day is optimal when taking these agents.

Pegloticase is a recombinant mammalian urate oxidase produced from a genetically modified Escherichia coli conjugated to multiple strands of monomethoxypoly ethyl glycol (PEG) [50]. Pegloticase, given as an intravenous infusion every 2 weeks, is typically reserved for patients with severe gout in whom target serum urate concentrations are not achieved or who cannot tolerate oral ULT [50]. Patients with contraindications to other ULT or who have large crystal burden are also candidates. Resistance can develop due to the development of anti-PEG antibodies, which can also increase the risk for adverse reactions. Pegloticase is typically prescribed by rheumatologists.

Treatment should also consider other commonly prescribed medications that can affect UA levels, including loop diuretics, hydrochlorothiazide, and fenofibrates. Loop and thiazide diuretics are known to increase uric acid reabsorption in the proximal tubule. Losartan has been shown to lower uric acid levels through inhibition of the renal urate transporter URAT1, thereby increasing urinary uric acid excretion and lowering UA [12]. Losartan can be substituted as an antihypertensive in place of a thiazide or loop diuretic where clinically appropriate. These medications, however, should not be discontinued when medically indicated. Fenofibrates can be used as an adjunct ULT when dyslipidemia is present $[26,32]$.

\section{CONCLUSIONS}

Gout is a debilitating disease of increasing worldwide prevalence and is associated with multiple comorbidities that leads to significant pain, disability, impaired quality of life, and societal costs. The silver lining is that gout is entirely curable, and through the use of a treat- to-target approach, it is possible to decrease MSU crystal deposition burden, prevent further crystal deposition, and subsequently eliminate the acute and chronic inflammation caused by gout. There are a number of pharmacologic agents that can be used alone or in combination to treat acute flares, and also to lower the concentration of SUA below the level of solubility. Despite all these tools at our disposal, gout remains a poorly controlled disease, and patients continue to suffer. Gout care can be improved dramatically through ongoing research, patient and provider education focusing on optimal management, and treating to a target SUA level of $<6 \mathrm{mg} / \mathrm{dl}(0.360 \mathrm{mmol} / \mathrm{l})$ or better.

\section{ACKNOWLEDGEMENTS}

Funding. No funding or sponsorship was received for this study or publication of this article.

Authorship. All named authors meet the International Committee of Medical Journal Editors (ICMJE) criteria for authorship for this article, take responsibility for the integrity of the work as a whole, and have given their approval for this version to be published.

Disclosures. Robert T. Keenan has been or is a consultant with Horizon Pharma, Selecta Biosciences, Sobi; and has received research support from Sobi. Jon Golenbiewski has nothing to disclose.

Compliance with Ethics Guidelines. This article is based on previously conducted studies and does not contain any studies with human participants or animals performed by any of the authors.

Open Access. This article is distributed under the terms of the Creative Commons Attribution-NonCommercial 4.0 International License (http://creativecommons.org/licenses/ by-nc/4.0/), which permits any noncommercial use, distribution, and reproduction in any 
medium, provided you give appropriate credit to the original author(s) and the source, provide a link to the Creative Commons license, and indicate if changes were made.

\section{REFERENCES}

1. Keenan RT. Limitations of the current standards of care for treating gout and crystal deposition in the primary care setting: a review. Clin Ther. 2017;39(2):430-41.

2. Robinson PC, Taylor WJ, Dalbeth N. An observational study of gout prevalence and quality of care in a national Australian general practice population. J Rheumatol. 2015;42(9):1702-7.

3. Roddy E, Packham J, Obrenovic K, Rivett A, Ledingham JM. Management of gout by UK rheumatologists: a British Society for Rheumatology national audit. Rheumatology (Oxford). 2018;57(5):826-30.

4. Khanna P, Khanna D, Storgard C, Baumgartner S, Morlock R. A world of hurt: failure to achieve treatment goals in patients with gout requires a paradigm shift. Postgrad Med. 2016;128(1):34-40.

5. Stamp LK, Merriman TR, Barclay ML, Singh JA, Roberts RL, Wright DF, et al. Impaired response or insufficient dosage? Examining the potential causes of "inadequate response" to allopurinol in the treatment of gout. Semin Arthritis Rheum. 2014;44(2):170-4.

6. Keenan RT, Krasnokutsky S, Pillinger MH. Etiology and pathogenesis of hyperuricemia and gout. In: Firestein GS, editor. Kelley and Firestein's textbook of rheumatology. II. Philadelphia: Elsevier; 2017. p. 1597-619.

7. Dalbeth N, Lindsay K. The patient's experience of gout: new insights to optimize management. Curr Rheumatol Rep. 2012;14(2):173-8.

8. Dalbeth N, Merriman TR, Stamp LK. Gout. Lancet. 2016;388(10055):2039-52.

9. Kuo CF, Grainge MJ, Zhang W, Doherty M. Global epidemiology of gout: prevalence, incidence and risk factors. Nat Rev Rheumatol. 2015;11(11):649-62.

10. Chen-Xu M, Yokose C, Rai SK, Pillinger MH, Choi HK. Contemporary prevalence of gout and hyperuricemia in the United States and decadal trends: the national health and nutrition examination survey 2007-2016. Arthritis Rheumatol. 2019. https://doi.org/10.1002/art.40807.

11. Smith E, Hoy D, Cross M, Merriman TR, Vos T, Buchbinder R, et al. The global burden of gout: estimates from the Global Burden of Disease 2010 study. Ann Rheum Dis. 2014;73(8):1470-6.

12. Stamp LK, Chapman PT. Gout and its comorbidities: implications for therapy. Rheumatology (Oxford). 2013;52(1):34-44.

13. Zhu Y, Pandya BJ, Choi HK. Prevalence of gout and hyperuricemia in the US general population: the National Health and Nutrition Examination Survey 2007-2008. Arthritis Rheum. 2011;63(10):3136-41.

14. Pagidipati NJ, Clare RM, Keenan RT, Chiswell K, Roe MT, Hess CN. Association of gout with longterm cardiovascular outcomes among patients with obstructive coronary artery disease. J Am Heart Assoc. 2018;7(16):e009328.

15. Major TJ, Topless RK, Dalbeth N, Merriman TR. Evaluation of the diet wide contribution to serum urate levels: meta-analysis of population based cohorts. BMJ. 2018;363:k3951.

16. Dalbeth N, Stamp LK, Merriman TR. The genetics of gout: towards personalised medicine? BMC Med. 2017;15(1):108.

17. Stamp L, Dalbeth N. Urate-lowering therapy for asymptomatic hyperuricaemia: a need for caution. Semin Arthritis Rheum. 2017;46(4):457-64.

18. Dalbeth N, Haskard DO. Mechanisms of inflammation in gout. Rheumatology (Oxford). 2005;44(9):1090-6.

19. Neogi T. Clinical practice. Gout. N Engl J Med. 2011;364(5):443-52.

20. Dalbeth N, Stamp L. Hyperuricaemia and gout: time for a new staging system? Ann Rheum Dis. 2014;73(9):1598-600.

21. Marson FG. Effect of ACTH and sodium salicylate on the urinary uric acid: creatinine ratio, and circulating eosinophils in man. Ann Rheum Dis. 1953;12(4):296-300.

22. Tennant F. The physiologic effects of pain on the endocrine system. Pain Ther. 2013;2(2):75-86.

23. Zhao T, Lv X, Cao L, Guo M, Zheng S, Xue Y, et al. Renal excretion is a cause of decreased serum uric acid during acute gout. Int $\mathrm{J}$ Rheum Dis. 2018;21(9):1723-7.

24. Urano $W$, Yamanaka $H$, Tsutani $H$, Nakajima $H$, Matsuda Y, Taniguchi A, et al. The inflammatory 
process in the mechanism of decreased serum uric acid concentrations during acute gouty arthritis. J Rheumatol. 2002;29(9):1950-3.

25. Khanna D, Khanna PP, Fitzgerald JD, Singh MK, Bae S, Neogi T, et al. 2012 American College of Rheumatology guidelines for management of gout. Part 2: therapy and antiinflammatory prophylaxis of acute gouty arthritis. Arthritis Care Res (Hoboken). 2012;64(10):1447-61.

26. Hui M, Carr A, Cameron S, Davenport G, Doherty $M$, Forrester $H$, et al. The British Society for Rheumatology guideline for the management of gout. Rheumatology (Oxford). 2017;56(7):1056-9.

27. Richette P, Doherty M, Pascual E, Barskova V, Becce F, Castaneda-Sanabria J, et al. 2016 updated EULAR evidence-based recommendations for the management of gout. Ann Rheum Dis. 2017;76(1):29-42.

28. Keenan RT, O'Brien WR, Lee KH, Crittenden DB, Fisher MC, Goldfarb DS, et al. Prevalence of contraindications and prescription of pharmacologic therapies for gout. Am J Med. 2011;124(2):155-63.

29. Dalbeth N, Lauterio TJ, Wolfe HR. Mechanism of action of colchicine in the treatment of gout. Clin Ther. 2014;36(10):1465-79.

30. Desmarais J, Chu CQ. Utility of anakinra in acute crystalline diseases: a retrospective study comparing a university hospital with a Veterans Affairs medical center. J Rheumatol. 2018. https://doi.org/10.3899/ jrheum.180393.

31. ClinicalTrials.gov. A study to evaluate efficacy and safety of anakinra in the treatment of acute gouty arthritis (anaGO): U.S. National Library of Medicine; 2016. https://clinicaltrials.gov/ct2/show/ NCT03002974. Accessed 19 Jan 2019.

32. Khanna D, Fitzgerald JD, Khanna PP, Bae S, Singh MK, Neogi T, et al. 2012 American College of Rheumatology guidelines for management of gout. Part 1: systematic nonpharmacologic and pharmacologic therapeutic approaches to hyperuricemia. Arthritis Care Res (Hoboken). 2012;64(10):1431-46.

33. Hill EM, Sky K, Sit M, Collamer A, Higgs J. Does starting allopurinol prolong acute treated gout? A randomized clinical trial. J Clin Rheumatol. $2015 ; 21(3): 120-5$.

34. Pascart T, Liote F. Gout: state of the art after a decade of developments. Rheumatology (Oxford). 2019;58(1):27-44.

35. Fields TR, Batterman A. How can we improve disease education in people with gout? Curr Rheumatol Rep. 2018;20(3):12.
36. Abhishek A, Doherty M. Education and non-pharmacological approaches for gout. Rheumatology (Oxford). 2018;57(suppl_1):i51-8.

37. Rai SK, Fung TT, Lu N, Keller SF, Curhan GC, Choi HK. The dietary approaches to stop hypertension (DASH) diet, Western diet, and risk of gout in men: prospective cohort study. BMJ. 2017;357:j1794.

38. Qaseem A, Harris RP, Forciea MA. Management of acute and recurrent gout: a clinical practice guideline from the American College of Physicians. Ann Intern Med. 2017;166(1):58-68.

39. Neogi T, Mikuls TR. To treat or not to treat (to target) in gout. Ann Intern Med. 2017;166:71-2.

40. Bursill D, Dalbeth N. What is the evidence for treatto-target serum urate in gout? Curr Rheumatol Rep. 2018;20(3):11.

41. Dalbeth N, Bardin T, Doherty M, Liote F, Richette P, Saag KG, et al. Discordant American College of Physicians and international rheumatology guidelines for gout management: consensus statement of the Gout, Hyperuricemia and Crystal-Associated Disease Network (G-CAN). Nat Rev Rheumatol. 2017;13(9):561-8.

42. Thiele RG, Schlesinger N. Ultrasonography shows disappearance of monosodium urate crystal deposition on hyaline cartilage after sustained normouricemia is achieved. Rheumatol Int. 2010;30(4):495-503.

43. Doherty M, Jenkins W, Richardson H, Sarmanova A, Abhishek A, Ashton D, et al. Efficacy and cost-effectiveness of nurse-led care involving education and engagement of patients and a treat-to-target urate-lowering strategy versus usual care for gout: a randomised controlled trial. Lancet. 2018;392(10156):1403-12.

44. Mikuls TR, Cheetham TC, Levy GD, Rashid N, Kerimian A, Low KJ, et al. A pharmacist-led intervention to improve gout medication adherence and outcomes with urate lowering therapy: a site randomized trial. Am J Med. 2018. https://doi.org/10. 1016/j.amjmed.2018.11.011.

45. Becker MA, Baraf HS, Yood RA, Dillon A, VazquezMellado J, Ottery FD, et al. Long-term safety of pegloticase in chronic gout refractory to conventional treatment. Ann Rheum Dis. 2013;72(9):1469-74.

46. Lipsky PE, Calabrese LH, Kavanaugh A, Sundy JS, Wright D, Wolfson M, et al. Pegloticase immunogenicity: the relationship between efficacy and antibody development in patients treated for refractory chronic gout. Arthritis Res Ther. 2014;16(2):R60. 
47. De Vera MA, Marcotte G, Rai S, Galo JS, Bhole V. Medication adherence in gout: a systematic review. Arthritis Care Res (Hoboken). 2014;66(10):1551-9.

48. Robinson PC, Dalbeth N, Donovan P. The cost-effectiveness of biannual serum urate (SU) monitoring after reaching target in gout: a health economic analysis comparing SU monitoring. J Rheumatol. 2018;45(5):697-704.

49. Stamp LK, Day RO, Yun J. Allopurinol hypersensitivity: investigating the cause and minimizing the risk. Nat Rev Rheumatol. 2016;12(4):235-42.

50. Keenan RT. Safety of urate-lowering therapies: managing the risks to gain the benefits. Rheum Dis Clin N Am. 2012;38(4):663-80.

51. Stamp LK, O'Donnell JL, Zhang M, James J, Frampton C, Barclay ML, et al. Using allopurinol above the dose based on creatinine clearance is effective and safe in patients with chronic gout, including those with renal impairment. Arthritis Rheum. 2011;63(2):412-21.

52. Stamp LK, Chapman PT, Barclay ML, Horne A, Frampton C, Tan P, et al. A randomised controlled trial of the efficacy and safety of allopurinol dose escalation to achieve target serum urate in people with gout. Ann Rheum Dis. 2017;76(9):1522-8.

53. Stamp LK, Chapman PT, Barclay $M$, Horne A, Frampton C, Tan P, et al. Allopurinol dose escalation to achieve serum urate below $6 \mathrm{mg} / \mathrm{dl}$ : an open-label extension study. Ann Rheum Dis. 2017;76(12):2065-70.

54. Stamp LK, Chapman PT, Barclay ML, Horne A, Frampton C, Tan P, et al. How much allopurinol does it take to get to target urate? Comparison of actual dose with creatinine clearance-based dose. Arthritis Res Ther. 2018;20(1):255.

55. Edwards NL. Febuxostat: a new treatment for hyperuricaemia in gout. Rheumatology (Oxford). 2009;48(Suppl 2):ii15-9.

56. Becker MA, Schumacher HR, Espinoza LR, Wells AF, MacDonald P, Lloyd E, et al. The urate-lowering efficacy and safety of febuxostat in the treatment of the hyperuricemia of gout: the CONFIRMS trial. Arthritis Res Ther. 2010;12(2):R63.

57. Schumacher HR Jr, Becker MA, Wortmann RL, Macdonald PA, Hunt B, Streit J, et al. Effects of febuxostat versus allopurinol and placebo in reducing serum urate in subjects with hyperuricemia and gout: a 28-week, phase III, randomized, double-blind, parallel-group trial. Arthritis Rheum. 2008;59(11):1540-8.

58. White WB, Saag KG, Becker MA, Borer JS, Gorelick $\mathrm{PB}$, Whelton A, et al. Cardiovascular safety of febuxostat or allopurinol in patients with gout. N Engl J Med. 2018;378(13):1200-10.

59. Choi H, Neogi T, Stamp L, Dalbeth N, Terkeltaub R. New perspectives in rheumatology: implications of the cardiovascular safety of febuxostat and allopurinol in patients with gout and cardiovascular morbidities trial and the associated food and drug administration public safety alert. Arthritis Rheumatol. 2018;70(11):1702-9.

60. Jansen TL, Perez-Ruiz F, Tausche AK, Richette P. International position paper on the appropriate use of uricosurics with the introduction of lesinurad. Clin Rheumatol. 2018;37(12):3159-65. 